\title{
Effects of Human Umbilical Cord Mesenchymal Stem Cells on Renal Ischaemia-reperfusion Injury in Rats
}

\author{
Zhenyu Qiu, Dun Zhou, Dongxiao Sun \\ Department of Nephrology, the First Affiliated Hospital, Liaoning Medical College, Jinzhou 121001, \\ China
}

\section{ABSTRACT}

Objective: This study aims to observe the function of umbilical cord-mesenchymal stem cells (UC-MSCs) labelled with enhanced green fluorescent protein (eGFP) in the repair of renal ischaemia-reperfusion (I/R) injury, to determine the effects on inflammatory cascade in an established rat model and to explore possible pathogenesis.

Materials and Methods: Sixty rats were randomly divided into three groups: the sham-operated, I/R and UC-MSC treatment groups. All rats underwent right nephrectomy. Ischaemia was induced in the left kidney by occlusion of the renal artery and vein for 1 hour, followed by reperfusion for 24 hours or 48 hours. Kidney samples were collected to observe morphological changes. Immunohistochemistry was performed to assess the expression of intercellular adhesion molecule 1 (ICAM-1) in the renal tissue sample, as well as the number of infiltrating polymorphonuclear neutrophils (PMNLs) and UC-MSCs with positive eGFP.

Results: Renal histopathological damages and the expression of ICAM-1 and PMNL increased significantly in the I/R group compared with those in the sham-operated group, whereas the damages were less conspicuous in the UC-MSC treatment group.

Conclusions: Renal ICAM-1, which mediated PMNL infiltration and contributed to renal damage, was significantly up-regulated in the I/R group. UC-MSCs were identified to inhibit these pathological processes and protect the kidney from I/R injury.

\section{ARTICLE INFO}

Key words:

Umbilical Cord; enhanced green fluorescent protein [Supplementary Concept]; Intercellular Adhesion Molecule-1

Int Braz J Urol. 2014; 40: 553-61

Submitted for publication:

September 14, 2013

Accepted after revision:

February 07, 2014

\section{INTRODUCTION}

Renal ischaemia-reperfusion (I/R) injury is commonly observed in clinic and is considered as the main cause of acute renal failure (ARF). Over the last few decades, blood purification technology and critical care medicine have significantly developed, but the mortality of acute tubular necrosis (ATN) induced by septicaemia, shock, and serious trauma did not decline, maintaining a range of 30\% - 50\% (1). More studies have focused on the promotion of the regeneration and repair of necrotic renal tubular epithelial cells and on the reduction of ARF mortality. With the improvement in stem cell research, a new method is proposed for the treatment of ischaemia-induced irreversible renal tubular damages based on the repair and regenerative capability of stem cells. Mesenchymal stem cells (MSCs) are adult stem cells present in different tissues of the body and are derived from the bone marrow $(2,3)$, peripheral blood $(4,5)$, umbilical cord blood $(6,7)$ and fat (8). Several studies have been performed on bone marrow MSCs (BM-MSCs). BM-MSCs have been used as seed cells 
in different fields of transplantation therapy, but umbilical cord mesenchymal stem cells (UC-MSCs) are rarely studied and are thus confronted with numerous unsolved problems. Compared with the adult bone marrow, the umbilical cord has numerous advantages, such as extensive source, easy collection and transformation of waste into beneficial products. The isolation of UC-MSCs from the whole umbilical cord provides an extensive and new source of MSCs, which can significantly promote clinical application.

To study the possibility of applying MSCs to the treatment of tubular injury disease, several researchers used an animal model of I/R to examine BM-MSCs. Results showed that BM-MSCs promote renal tubular damage repair and protect renal function. Moreover, BM-MSCs can differentiate into renal tubular epithelial cells and vascular endothelial cells, which are directly involved in the repair of renal tubular injury (9). In addition, BM-MSCs can improve and promote endogenous renal proliferation repair, which depends on the complex-regulated paracrine mechanism $(10,11)$. However, the repair mechanisms of BM-MSCs vary in different laboratories, and the possibility that UC-MSC mechanisms are identical to those of BM-MSCs is rarely investigated.

The inflammatory cascade induced by $I / R$ is an important factor causing renal injury. Vascular endothelial cell dysfunction with high expression of intercellular adhesion molecule-1 (ICAM1) and activated white blood cells (WBCs) and tissue macrophages can initiate the inflammatory cascade (12). After transplantation, MSCs hypothetically enter the blood circulation and express various adhesion molecules, such as ICAM-1, vascular cell adhesion molecule-1 (VCAM-1), L-selectin and P-selectin. The neutrophils are activated, reach the specific damage sites and adhere to the vascular endothelium. These neutrophils enter the renal interstitium through the vessels with increased permeability, partially replace the original infiltration site of the neutrophils and reduce neutrophil infiltration. This mechanism reduces the damage induced by reactive oxygen species, inflammatory mediators and proteases in the tissue at the acute injury stage. No direct experimental evidence confirms the possibility that UC-MSCs express similar factors, directly differentiate into renal tubular epithelial cells or vascular endothelial cells, or promote endogenous repair either through inflammatory response reduction or with the combination of the two mentioned pathways.

In this study, caudal vein injection of UC-MSCs was performed to treat rats with renal I/R. The repair function of UC-MSCs and their effects on inflammatory cascade after I/R were observed to elucidate the UC-MSC plasticity and enhance the theory of acute tubular necrosis repair. ICAM1 expression in the renal tissue and WBC infiltration, and their relationship with UC-MSC homing number, were detected. Moreover, the effect of UC-MSC homing on the inflammatory cascade and on the renal protection mechanism was preliminarily investigated.

\section{MATERIALS AND METHODS}

\section{Animals and grouping}

Twenty healthy male Sprague-Dawley (SD) rats with body weight ranging from $250 \mathrm{~g}$ to $300 \mathrm{~g}$ were provided by the Laboratory Animal Centre of Jinzhou Medical College. After adaptive breeding for 7 days, the rats with negative urine screening results were randomized into three groups: the sham-operated (sham), I/R and human umbilical cord mesenchymal stem cell (UC$-\mathrm{MSC}+\mathrm{I} / \mathrm{R})$ groups. Each group had 20 rats. This study was carried out in strict accordance with the recommendations in the Guide for the Care and Use of Laboratory Animals of the National Institutes of Health. The animal use protocol has been reviewed and approved by the Institutional Animal Care and Use Committee (IACUC) of Liaoning Medical College.

Establishment of rat model of renal $I / R$ and sampling process

The rats in the I/R group were anaesthetized intraperitoneally with 20\% urethane. The left renal pedicle was isolated and occluded by non-invasive artery clamp for 1 hour. The clamp was then removed to monitor the recovered renal blood flow. The indication of successful reperfusion is the change in the renal colour from dark red (resulting from ischaemia) to bright red within $5 \mathrm{~min}$. 
of artery clamp removal. The right kidney was removed, and abdomen closure was performed layer by layer. In the UC-MSC group, $1 \times 10^{6}(0.5 \mathrm{~mL})$ UC-MSCs transfected with enhanced green fluorescent protein (eGFP-UCMSCs) (Heze Biotech Co., Ltd., Beijing, China) were injected into the caudal vein of the rats $30 \mathrm{~min}$. after reperfusion. In the sham group, the right kidney of the rats was removed, and the left renal pedicle was isolated but not occluded. The same volume of normal saline was injected into the caudal vein of the rats in the $\mathrm{I} / \mathrm{R}$ and sham groups. Ten rats from each group were executed 24 and 48 hours after injection. The left kidney (approximately three quarters) from each rat was fixed in FAA solution (every $100 \mathrm{~mL}$ of FAA solution contained $10 \mathrm{~mL}$ of formalin, $5 \mathrm{~mL}$ of glacial acetic acid and $85 \mathrm{~mL}$ of $95 \%$ ethanol) for further research, and the rest of the tissue was prepared for conventional frozen section.

\section{Histological study}

The renal tissue samples were cut into $2 \mu \mathrm{m}$ slices after fixation in FAA solution, dehydration and paraffin embedding. Hematoxylin and eosin (HE) staining was performed on the slides, and the lesions were examined using a light microscope. Paller's criterion for grading tubulointerstitial lesion was applied. To record the score, 10 renal tubules were randomly selected from every high-power field. The tubule with apparent expansion and flat or swelling cells scored one point, whereas those with a brush border were injured or fell off and scored one or two points. Moreover, urinary casts scored two points, the necrotic cells (did not form cast or fragments) in the tubule cavity scored one point. The scoring process was performed in 10 random fields (i.e., 100 renal tubules).

\section{Leukocyte counting}

Inflammatory cells (per mm2) that infiltrated into the tubule interstitium were analysed by using a computerized medical image analysis system (CMIAS), which indicated the amount of leukocytes in the renal tissue.

\section{ICAM-1 detection \\ Rabbit-anti-rat ICAM-1 and SP working solution kit (Wuhan Boster Biological Enginee-}

ring Co., Ltd., Wuhan, China) were applied to detect the ICAM-1 expression in the renal tissue by immunohistochemistry. The $4 \mu \mathrm{m}$ tissue sections underwent routine deparaffinization and rehydration. Freshly prepared 3\% hydrogen peroxide was used to deactivate endogenous peroxidase. After antigen retrieval by heat, protein block solution was added to block the sample for $20 \mathrm{~min}$. The slides were incubated with rabbit-anti-rat ICAM-1 antibody overnight at $4^{\circ} \mathrm{C}$ and with biotinylated secondary antibody at room temperature (RT) for 20 min. Strept Avidin-Biotin Complex was employed at RT for 20 min., followed by DAB developing. The developing time was controlled under a microscope. Finally, hematoxylin was applied for counterstaining, followed by dehydration, transparency and mounting. The negative control received PBS instead of the primary antibody. Results were determined by claybank particles that indicated a positive site. The dark brown area was the strong positive, claybank was positive and light yellow was weak positive. The uncoloured cells were considered negative cells.

\section{Pathological image analysis}

An inverted diffraction phase and fluorescence microscope (Leica Company, Berlin, Germany) was used to select 10 high power fields randomly at the lesion site on immunohistochemical slides from each group. CMIAS multi-functional true colour pathological image analysis system (Motic Image Technology Co., Ltd, Beijing, China) was employed, and computerized image analysis software facilitated computer reading to recognize the immunohistochemically positive signals of the selected slide fields. The expression of ICAM-1 in the renal tissue was assessed semi-quantitatively by calculating the integration of positive-coloured area. This result represented the integrated optical density value for each field, and the average of these 10 values represented the value for each sample. Finally, the values were compared among the groups.

The renal tissue samples were embedded with OCT and cut into $10 \mu \mathrm{m}$ slides. An inverted diffraction phase and fluorescence microscope was used to observe the GFP expression in cells of the renal tissue in each group when the slide was ex- 
posed to UV (488 $\pm 15 \mathrm{~nm})$. The green cells were the positive eGFP-UCMSCs after transplantation. The number of GFP-positive cells was counted in five high-power fields (400x) for each slide. The percentage of positive cells was calculated as: positive cell $\%=$ number of positive cells/ (number of positive and negative cells) x 100\%.

\section{Statistical analysis}

All values were presented as mean \pm standard deviation after quantitative or semi-quantitative analysis and then subjected to one-factor analysis of variance using SPSS 13.0 software. The linear correlation analysis of indices was employed. Differences were considered significant at $\mathrm{P}<0.05$.

\section{RESULTS}

Histopathological changes of renal tissue in rats Renal tubules were arranged neatly in the sham group, and no congestion edema was observed in the mesenchyme (Figure-1A). The renal cortex and medulla in the $I / R$ group were observed to have tinted white colour with mild swelling. Result of HE staining under a microscope showed the proximal convoluted tubules in an inordinate and loose arrangement. Epithelial cells apparently swelled and were characterized by an extensive vacuolar degeneration and flaky necrosis. The nuclei of minor epithelial cells were discovered in pyknosis and deep dyeing, and epithelium cells fell off the lumen. Large urinary casts were initially formed in distal convoluted tubules. Renal interstitium was locally bled and complicated with inflammatory cell infiltration. Apparent injury was detected in the $\mathrm{I} / \mathrm{R} 24 \mathrm{~h}$ group (Figure-1B), that was worse in the I/R 48 hours group (Figures $1 \mathrm{C}$ and D). The UC-MSC group showed light tissue injury, mild edema of renal interstitium and normal arrangement of renal tubules. Mild swelling of tubular epithelial cells in areas where brush border fell off and urinary casts were occasionally observed. Inflammatory cell infiltration was also less serious in the UC-MSC group compared with the $\mathrm{I} / \mathrm{R}$ groups (Figures 1E, F and G). The changes in all groups were more severe after 48 hours than after 24 hours.

\section{Figure 1 - HE staining of renal tissue.}
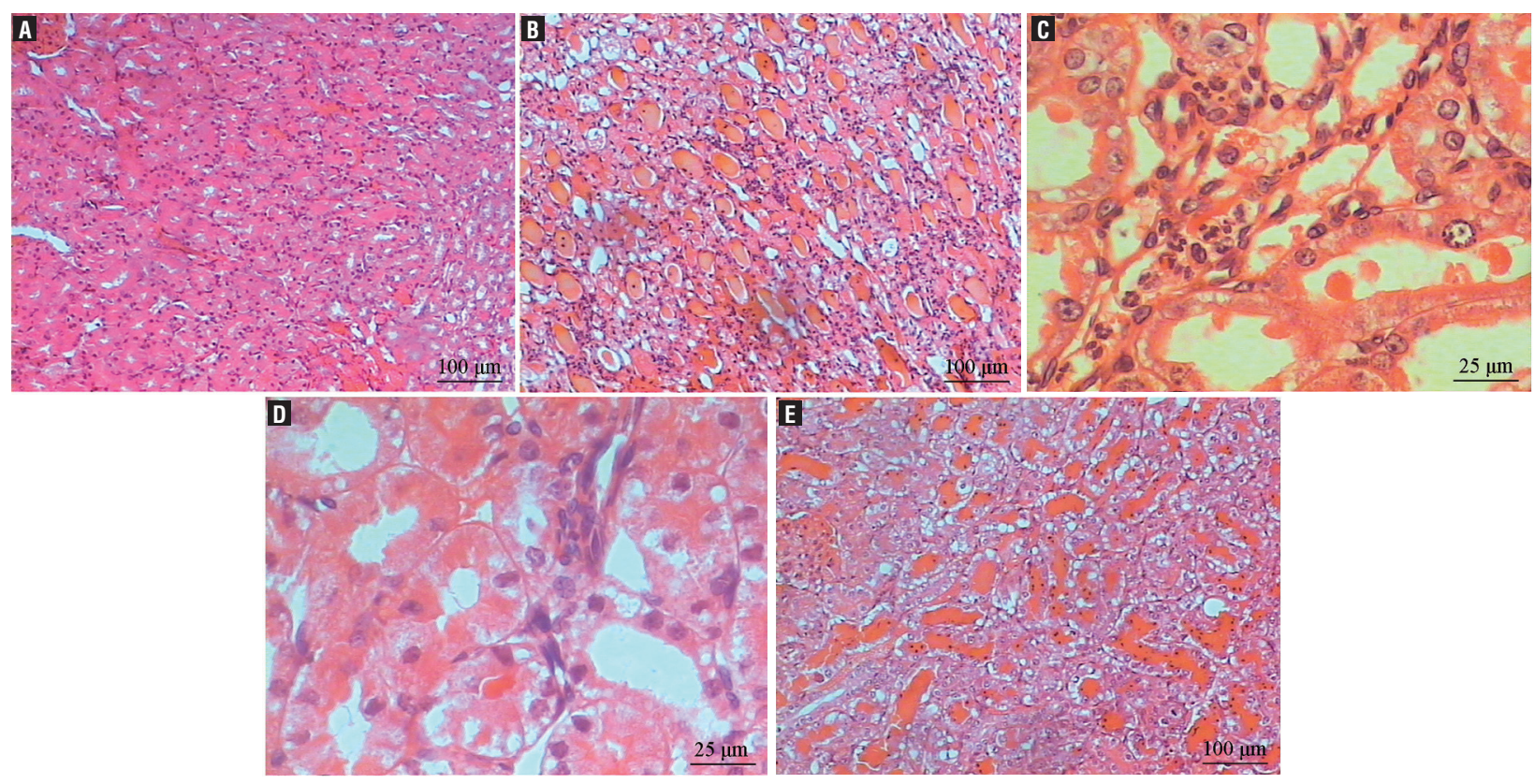

A) the sham-operated group (100x); B) the I/R group at $24 \mathrm{~h}(400 \mathrm{x}) ; \mathbf{C})$ the I/R group at 48 hours (100x); D) the I/R group at 48 hours (400x); E) the UC-MSCs+I/R group at 24 hours (400x); F) the UC-MSCS+I/R at 48 hours (400x); G) the UC-MSCs+I/R at 48 hours (100x). 
Scoring of renal tubule and leukocyte counting in renal tissue

Single factor analysis of variance between groups was used for the pair-wise comparison of the renal tubular score among different groups. Results showed that compared with those in the sham group, the renal tubular score and leukocyte number in the renal tissue in $\mathrm{I} / \mathrm{R}$ group increased significantly $(\mathrm{P}<0.01)$. The renal tubular score and leukocyte number in renal tissue in the UC-MSC group were significantly lower than that in the I/R group $(\mathrm{P}<0.01)$. Significant differences were observed after 24 and 48 hours between the other two groups $(\mathrm{P}<0.05)$. Data are summarized in Table-1.

\section{ICAM-1 expression in renal tissue}

The sham group showed trace ICAM1 expression in the renal tissue in Figure-2A. ICAM-1 expression was prominent in the renal vasa recta, peritubular capillaries, glomerulus, proximal convoluted tubule and renal interstitium, which was significantly up-regulated within 48 hours. The expression of ICAM-1 (Figure-2B) in the I/R $24 \mathrm{~h}$ group was higher in the $\mathrm{I} / \mathrm{R} 48$ hours group $(\mathrm{P}<0.01)$ (Figure-2C). ICAM-1 expression was significantly lower in the UC-MSC group than that in the I/R group (P $<0.01$ ) (Figure-2D). Significant differences in ICAM-1 expression were observed between 24 and 48 hours in all groups $(\mathrm{P}<0.01)$ (Figure$-2 \mathrm{E})$, except for the sham group (Table-2).
Ratio of positive eGFP-UCMSCs

A fluorescent microscope revealed no GFP-positive UC on the frozen sections of the kidney in the sham and $\mathrm{I} / \mathrm{R}$ groups (Figures $3 \mathrm{~A}$ and B). GFP-positive MSCs were found among the renal tubule cells in the UC-MSC group (Figures $3 \mathrm{C}$ and D), and the presence of GFP-positive MSCs was higher in the $48 \mathrm{~h}$ group than in the $24 \mathrm{~h}$ group $(\mathrm{P}<0.01)$. Data are shown in Table-3.

\section{Correlation analysis}

Using linear correlation analysis, the three groups of data were combined to analyze the correlation among the four detected indices. The correlation study of the renal tubule score, leukocyte number in renal tissue and ICAM-1 expression showed that renal tubule score of 24 and 48 hours reperfusion was positively correlated with the leukocyte number in renal tissue and ICAM-1 expression ( $\mathrm{r}=0.89,0.88$, respectively, $\mathrm{P}<0.01$ ). The renal tubule score, leukocyte number in renal tissue and ICAM-1 expression in the renal tissue positively correlated with the ratio of positive eGFP-UCMSCs $(\mathrm{r}=0.73, \mathrm{P}<0.01)$.

\section{DISCUSSION}

In this study, caudal vein injection with UC-MSCs was performed for the treatment of renal I/R rats. The rate of eGFP-UCMSC positive cells in the renal tissues and ICAM-1 expression were observed, and the effect of UCMSCs on I/R renal repair and

Table 1 - Renal tubule scoring and renal tissue leukocyte counting in different groups.

\begin{tabular}{|c|c|c|c|c|}
\hline \multirow[t]{2}{*}{ Group } & \multicolumn{2}{|c|}{ Score of renal tubule } & \multicolumn{2}{|c|}{ Leukocyte in renal tissue $\left(\mathrm{n} / \mathrm{mm}^{2}\right)$} \\
\hline & 24 hours & 48 hours & 24 hours & 48 hours \\
\hline Sham & $1.40 \pm 1.17$ & $1.40 \pm 1.07$ & $0.90 \pm 1.10$ & $1.30 \pm 1.77$ \\
\hline I/R & $169.60 \pm 15.68^{\#}$ & $182.40 \pm 12.77 \#$ & $39.20 \pm 11.99 \#$ & $66.70 \pm 15.51 \#$ \\
\hline UC-MSCs & $71.90 \pm 16.26^{\#^{*}}$ & $90.20 \pm 15.01^{\#^{*}}$ & $18.90 \pm 7.45^{\#^{\star}}$ & $27.20 \pm 9.53 \#^{*}$ \\
\hline
\end{tabular}

Note: $\# \mathrm{P}<0.01$ vs. sham group; ${ }^{*} \mathrm{P}<0.01 \mathrm{vs}$. I/R group. 
Figure 2 - Expression of ICAM-1 in renal tissue detected by immunohistochemistry (100x).

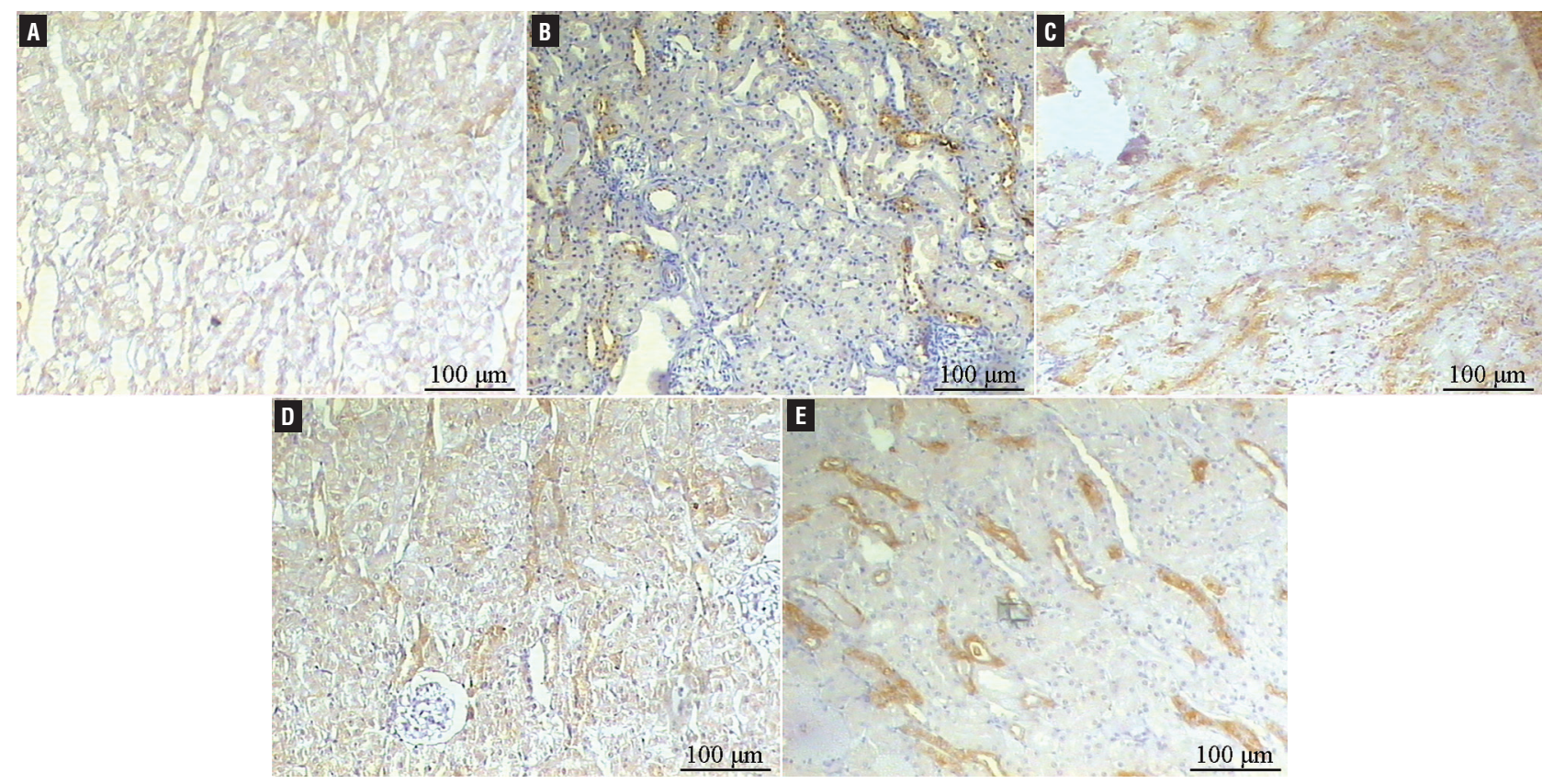

A) The sham-operated group; B) The I/R group at 24 hours; C) The I/R group at 48 hours; D) The UC-MSCs+1/R group at 24 hours; E) The UC-MSCs+I/R group at 48 hours.

Table 2 - ICAM-1expression in renal tissue at different time in all groups.

\begin{tabular}{lccc}
\hline Group & N & 24 hours & 48 hours \\
\hline Sham & 10 & $0.2170 \pm 0.1063$ & $0.1919 \pm 0.0876$ \\
I/R & 10 & $5.2569 \pm 0.7397^{\#}$ & $8.1012 \pm 1.6640^{\#}$ \\
UC-MSCs & 10 & $2.2249 \pm 0.9637^{* *}$ & $4.4056 \pm 0.9802^{*^{*}}$ \\
\hline
\end{tabular}

Note: $\# \mathrm{P}<0.01$ vs. sham group; ${ }^{\star} \mathrm{P}<0.01$ vs. I/R group.

inflammatory cascade after I/R were investigated. Results were theoretically consistent.

In addition, eGFP-UCMSCs were transplanted to the rat model of renal $\mathrm{I} / \mathrm{R}$ injury and the homing condition was observed. The GFP that is isolated from jellyfish and stably expressed in mammalian cells is a luminescent protein. This protein has gained considerable attention because of its advantages, such as harmlessness to cell, strongest marker intensity among all marker techniques, no requirement for substrate during detection and long maintenance of fluorescence $(13,14)$. Results showed positive eGFP cells in the renal tissue of UC-
-MSCs 24 hours group, and the increase in positive eGFP cells was more significant after 48 hours in the UC-MSC group $(\mathrm{P}<0.01)$, which was positively correlated with the ratio of positive eGFP cells. Therefore, we speculated that UC-MSCs transplanted in the rat model of renal $\mathrm{I} / \mathrm{R}$ injury were similar to the neutrophils recruited by ICAM-1. These neutrophils adhered to the vascular endothelium in the specific sites of injury and infiltrated into renal interstitium through blood vessels with enhanced permeability. UC-MSCs inactivated part of activated ICAM-1 with homing and replaced the infiltration sites of neutrophils (15). Hence, neutrophil infiltration and renal 
Figure 3 - Green fluorescent protein-marked expression of UC-MSCs in the renal tissue (400x).

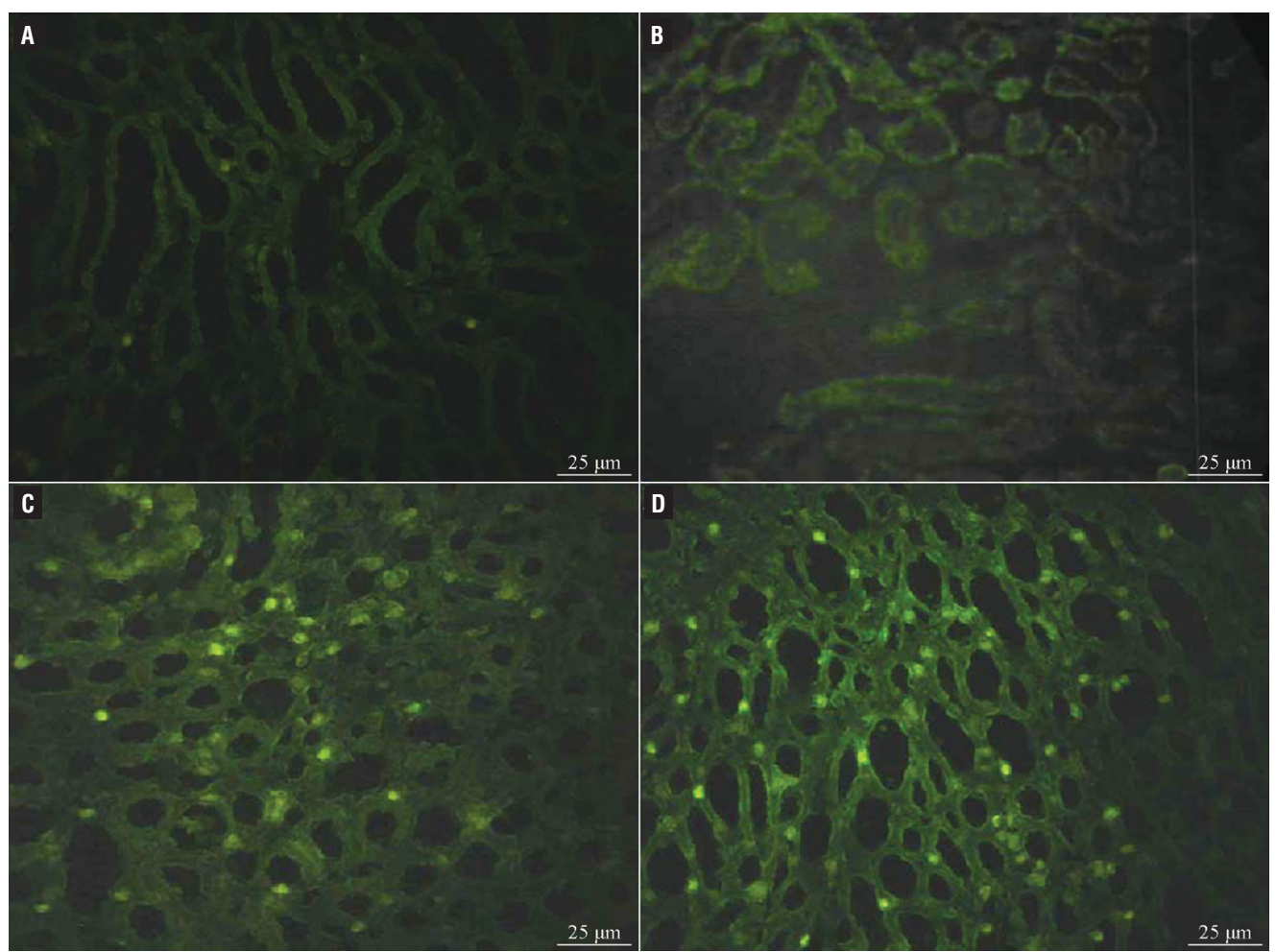

A) The sham-operated group; B) The I/R group at 48 hours; C) The UC-MSCs+I/R group at 24 hours; $\mathbf{D})$ The UC-MSCS+I/R group at 48 hours.

Table 3 - Ratio of positive eGFP-UCMSCs in renal tissue at different time in all groups (\%).

\begin{tabular}{lccc}
\hline Group & N & 24 hours & 48 hours \\
\hline Sham & 10 & 0 & 0 \\
I/R & 10 & 0 & 0 \\
UC-MSCs & 10 & $9.8 \pm 1.2^{*}$ & $21.2 \pm 7.3 \#^{*}$ \\
\hline
\end{tabular}

Note: $\# p<0.005$ vs. sham and I/R group; ${ }^{*} p<0.01$ UC-MSCs+I/R 48 hours vs. UC-MSCs+I/R 24 hours I/R group.

injury were decreased, and endogenous repair was promoted by alleviating the inflammatory cascade.

Results of this study showed that ICAM1 expression in the renal tissue in the $\mathrm{I} / \mathrm{R}$ group increased significantly. The infiltration degree of inflammatory cells was positively correlated with ICAM-1 expression, and the renal tubular score was also positively correlated to inflammatory cell infiltration degree. This finding indicated that up- -regulation of ICAM-1 expression and mediated leukocyte infiltration and recruitment are the key factors in renal $I / R$ that facilitate the occurrence and development of $\mathrm{I} / \mathrm{R}$ damage.

In this experiment, ICAM-1 expression and neutrophil infiltration remarkably declined in the UC-MSC group. UC-MSC homing might be regulated by multiple cytokines, including ICAM-1 expression, which were damaged by $\mathrm{I} / \mathrm{R}$. Togel et 
al. (11) treated the kidney of I/R rats by BM-MSC transplantation and achieved improvement in inflammation, vessels and necrotic renal tissue. The function of UC-MSCs is similar to that of BM-MSCs (16). A large number of studies show that UC-MSCs also express multiple receptor molecules, including VCAM-1, L-selectin, P-selectin and platelet endothelial cell adhesion molecule (PECAM) $(17,18)$, and that the ICAM-1 expression in MSCs requires induction. These receptor molecules interact with the highly expressed ICAM-1 by through the renal tubule under I/R conditions, resulting in the proliferation and homing of MSCs (19).

MSCs are adult stem cells that distribute in different tissues of body and are prominent in the bone marrow and UC (2). Considerable attention has been focused on bone marrow-derived MSCs, which have been applied to transplantation therapy as seed cells. However, few studies were reported on the treatment of renal I/R with UC-MSCs (20). According to a number of laboratory reports, the surface antigens of UC-MSCs have no specificity and possess strong self-renewal potency, high proliferation and multi-directional differentiation potential, which differentiate these antigens into any of the three germ layers and define them as the seed cells of various tissues (21). Previous studies on the effects of transplanting MSCs in renal $\mathrm{I} / \mathrm{R}$ have focused on the function of MSCs in renal colonization and differentiation (22).

The inflammatory cascade induced by renal $I / R$ is an important factor leading to renal damage (23). Vascular endothelial cell dysfunction, high expression of ICAM-1 and activation of leukocytes and macrophages may initiate inflammatory cascade (24). Numerous experiments indicate that adhesion and activation of neutrophils is the basis of I/R injury, which further causes mechanical obstruction, release of abundant inflammatory substances, activates more inflammatory cells and parenchyma cells and forms inflammatory cascade. Inhibiting leukocyte activation and blood vessel infiltration is the solution to reduce I/R injury, and blocking any link helps to ameliorate the $I / R$ injury $(25,26)$.

In this study, UC-MSC homing was preliminarily investigated. Results showed that UC-MSC homing and paracrine regulation may re- duce inflammation and improve and promote the endogenous renal proliferation. Research on UC-MSC has improved and has provided a new strategy for the treatment of renal tubular damages induced by I/R. However, many questions require further studies to be answered. For instance, in the application of UC-MSCs to the treatment of renal diseases, the consequences of UC-MSC receptor transplantation and its mechanism, as well as chromosome instability and risk of malignancy induced by in vitro UC-MSC amplification in long term (27), remain poorly understood. Therefore, more studies are needed to elucidate the biology of UC-MSCs in vivo and in vitro.

In conclusion, the multilineage differentiation capacity of MSCs can repair the renal tissue. Moreover, inflammatory cytokine consumption and inflammatory cell reduction during the MSC homing process contribute to the reduction of renal I/R injury and promote renal repair. The transplantation of MSCs may be an effective method for the treatment of severe renal tubular injury.

\section{ABBREVIATIONS}

BM-MSCs = bone marrow MSCs

eGFP = enhanced green fluorescent protein eGFP-UCMSCs = UC-MSCs transfected with enhanced green fluorescent protein

ICAM-1 = intercellular adhesion molecule 1

$\mathrm{I} / \mathrm{R}=$ renal ischaemia-reperfusion

MSCs = mesenchymal stem cells

PMNLs = polymorphonuclear neutrophils

UC-MSCs = umbilical cord-mesenchymal stem cells

\section{CONFLICT OF INTEREST}

None declared.

\section{REFERENCES}

1. Rabb H, O'Meara YM, Maderna P, Coleman P, Brady HR: Leukocytes, cell adhesion molecules and ischemic acute renal failure. Kidney Int. 1997; 51: 1463-8.

2. Bieback K, Kern S, Kocaömer A, Ferlik K, Bugert P: Comparing mesenchymal stromal cells from different human tissues: bone marrow, adipose tissue andumbilical cord blood. Biomed Mater Eng. 2008; 18: S71-6. 
3. Pittenger MF, Mackay AM, Beck SC, Jaiswal RK, Douglas R, Mosca JD, et al.: Multilineage potential of adult human mesenchymal stem cells. Science. 1999; 284: 143-7.

4. Zvaifler NJ, Marinova-Mutafchieva L, Adams G, Edwards CJ, Moss J, Burger JA, et al.: Mesenchymal precursor cells in the blood of normal individuals. Arthritis Res. 2000; 2: 477-88.

5. Umezawa A, Makino H: Cell source for regenerative medicine. Nihon Rinsho. 2008; 66: 865-72.

6. Erices $\mathrm{A}$, Conget $\mathrm{P}$, Minguell JJ: Mesenchymal progenitor cells in human umbilical cord blood. Br J Haematol. 2000; 109: 235-42.

7. Secco M, Zucconi E, Vieira NM, Fogaça LL, Cerqueira A, Carvalho MD, et al.: Multipotent stem cells from umbilical cord: cord is richer than blood! Stem Cells. 2008; 26: 146-50.

8. Safford KM, Hicok KC, Safford SD, Halvorsen YD, Wilkison WO, Gimble JM, et al.: Neurogenic differentiation of murine and human adipose-derived stromal cells. Biochem Biophys Res Commun. 2002; 294: 371-9.

9. Lin F, Cordes K, Li L, Hood L, Couser WG, Shankland SJ, et al.: Hematopoietic stem cells contribute to the regeneration of renal tubules after renal ischemia-reperfusion injury in mice. J Am Soc Nephrol. 2003; 14: 1188-99.

10. Duffield JS, Park KM, Hsiao LL, Kelley VR, Scadden DT, Ichimura T, et al.: Restoration of tubular epithelial cells during repair of the postischemic kidney occurs independently of bone marrow-derived stem cells. J Clin Invest. 2005; 115: 1743-55.

11. Tögel F, Hu Z, Weiss K, Isaac J, Lange C, Westenfelder C: Administered mesenchymal stem cells protect against ischemic acute renal failure through differentiationindependent mechanisms. Am J Physiol Renal Physiol. 2005; 289: F31-42.

12. Zhang XL, Selbi W, de la Motte C, Hascall V, Phillips A: Renal proximal tubular epithelial cell transforming growth factorbeta1 generation and monocyte binding. Am J Pathol. 2004; 165: 763-73.

13. Tsien RY: The green fluorescent protein. Annu Rev Biochem. 1998; 67: 509-44.

14. Flynn A, Barry F, O'Brien T: UC blood-derived mesenchymal stromal cells: an overview. Cytotherapy. 2007; 9: 717-26.

15. Gordon D, Glover CP, Merrison AM, Uney JB, Scolding NJ: Enhanced green fluorescent protein-expressing human mesenchymal stem cells retain neural marker expression. $J$ Neuroimmunol. 2008; 193: 59-67.

16. Liu Y, Dulchavsky DS, Gao X, Kwon D, Chopp M, Dulchavsky $S$, et al.: Wound repair by bone marrow stromal cells through growth factor production. J Surg Res. 2006; 136: 336-41.

17. Jiang ZS, Gao Y, Mu N: Multipotent adult progenitor cells from human bone marrow differentiate into hepatocyte-like cells induced by co-culture with human hepatocyte line. Zhonghua Yi Xue Za Zhi. 2007; 87: 414-8.
18. Le Blanc K, Tammik L, Sundberg B, Haynesworth SE, Ringdén 0: Mesenchymal stem cells inhibit and stimulate mixed lymphocyte cultures and mitogenic responses independently of the major histocompatibility complex. Scand J Immunol. 2003; 57: 11-20.

19. Gupta S, Verfaillie C, Chmielewski D, Kim Y, Rosenberg ME: A role for extrarenal cells in the regeneration following acute renal failure. Kidney Int. 2002; 62: 1285-90.

20. Sarugaser R, Lickorish D, Baksh D, Hosseini MM, Davies JE: Human umbilical cord perivascular (HUCPV) cells: a source of mesenchymal progenitors. Stem Cells. 2005; 23: 220-9.

21. Lee OK, Kuo TK, Chen WM, Lee KD, Hsieh SL, Chen TH: Isolation of multipotent mesenchymal stem cells from umbilical cord blood. Blood. 2004; 103: 1669-75.

22. Kern S, Eichler H, Stoeve J, Klüter H, Bieback K: Comparative analysis of mesenchymal stem cells from bone marrow, umbilical cord blood, or adipose tissue. Stem Cells. 2006; 24: 1294-301.

23. Singbartl K, Ley K: Leukocyte recruitment and acute renal failure. J Mol Med (Berl). 2004; 82: 91-101.

24. Baer PC, Geiger H: Different effects of growth factors on human renal early distal tubular cells in vitro. Kidney Blood Press Res. 2006; 29: 225-30.

25. Rentsch M, Post S, Palma P, Lang G, Menger MD, Messmer K: Anti-ICAM-1 blockade reduces postsinusoidal WBC adherence following cold ischemia and reperfusion, but does not improve early graft function in rat liver transplantation. J Hepatol. 2000; 32: 821-8.

26. Souza-Moraes MR, David-Filho R, Baptista-Silva JC, Ullian M, Franco MF, Gabriel A Jr, et al.: Effect of antibodies to intercellular adhesion molecule type 1 on the protection of distant organs during reperfusion syndrome in rats. Braz J Med Biol Res. 2003; 36: 605-12.

27. Bobick BE, Tuan RS, Chen FH: The intermediate filament vimentin regulates chondrogenesis of adult human bone marrow-derived multipotent progenitor cells. J Cell Biochem. 2010; 109: 265-76. 